\title{
Critical comparison of INAA and ICP-MS applied in the characterization of purity of TRISO fuel and substrates to its production
}

Ewelina Chajduk, Paweł Kalbarczyk

\begin{abstract}
The application of inductively coupled plasma mass spectrometry (ICP-MS), both in solution and laser ablation (LA) mode, and instrumental neutron activation analysis (INAA) in the nuclear material analysis are presented in this paper. The possibility of each technique for the chemical characterization of substances used during TRISO fuel production and its advantages and limitations are discussed based on the obtained results of the analysis of real materials used in TRISO fuel production in the Institute of Nuclear Chemistry and Technology. The paper also reports the application of INAA and LA-ICP-MS to the verification of the purity of the protective layers of pyrolytic carbon $(\mathrm{PyC})$ and silicon carbide.
\end{abstract}

Keywords: Chemical purity • Inductively coupled plasma mass spectrometry $\bullet$ Instrumental neutron activation analysis • TRISO coated fuel

E. Chajduk ${ }^{\bowtie}$, P. Kalbarczyk

Institute of Nuclear Chemistry and Technology

Laboratory of Nuclear Analytical Methods

Dorodna 16 Str., 03-195 Warsaw, Poland

E-mail: e.chajduk@ichtj.waw.pl

Received: 9 February 2021

Accepted: 12 April 2021

\section{Introduction}

High-temperature gas-cooled reactor (HTGR) is an advanced type of nuclear reactor. Nuclear reactors of this type are gaining more and more interest mainly due to their high thermal efficiency, higher safety level, and cost effectiveness [1-4]. Nuclear fuel used in modern HTGRs consists of spherical uranium dioxide particles covered with a very durable coating consisting of porous pyrolytic carbon (PyC), dense $\mathrm{PyC}$, silicon carbide, and the outer layer of dense PyC. This structure of the fuel with the TRi-structural ISOtropic (TRISO) coating guarantees that the fission products are retained inside the spherical particle, thus preventing the release of activity. TRISO coated nuclear fuel must adhere to very strict quality standards when it comes to the chemical composition of the individual layers. It should be emphasized that nuclear fuel, independently of the type of reactor, must fulfill several criteria. One of them is its purity. To ensure and control the high purity of nuclear fuel, it is necessary to have analytical procedures that can determine the trace and ultratrace amounts of impurities in a wide range of materials. Under certain conditions, even a small amount of impurities can alter the chemical and nuclear properties of a material; such alterations may cause corrosion, or could result in higher radiological hazards for the operation of reactors. From the analytical point of view, it would be most advantageous to have at least two different analytical techniques, based on differ-

0029-5922 @ 2021 The Author(s). Published by the Institute of Nuclear Chemistry and Technology.

This is an open access article under the CC BY-NC-ND 4.0 licence (http://creativecommons.org/licences/by-nc-nd/4.0/). 
ent physicochemical bases, which would allow: (1) routine analytical activities based on application of first analytical technique; and (2) verification of the developed procedures and the obtained results with the use of the second technique. Both instrumental neutron activation analysis (INAA) and inductively coupled plasma mass spectrometry (ICP-MS) are widely used in nuclear materials analysis [5-9].

The accurate determination of trace and ultratrace elements is recognized as a combination of sample digestion and measurement of given analyte. Sample dissolution/digestion is usually first step of the analytical procedures and has a significant impact on the final result of determinations. Depending on the sample material and its origin, different digestion methods are considered to be effective. In the case of materials that are difficult to digest, it is believed that the recovery of $90 \%$ is sufficient for analytical purposes. In the case of materials used in widely understood "nuclear reactor industry", it is necessary to obtain total digestion of analysed materials. For these materials, obtaining a total, quantitative digestion is crucial for having reliable analytical information, which is essential for nuclear safety.

Neutron activation analysis is a leading multielemental analytical technique used in the determination of elements at trace and ultratrace levels in various materials. Approximately $70 \%$ of the elements have nuclear properties suitable for NAA. In INAA, the elemental determination is based on the conversion of stable nuclei to other, mostly radioactive nuclei via nuclear reactions ( $\mathrm{AX}+\mathrm{n} \rightarrow$ (radioactive nucleus) $* \rightarrow{ }_{Z}^{A} \mathrm{Y}+b$ ), where: $\mathrm{X}$ - chemical symbol of the target element; $\mathrm{A}$ and $\mathrm{Z}$ - mass number and atomic number of the target element, respectively; $\mathrm{Y}$ - chemical symbol of the reaction product; $A^{\prime}$ and Z' - mass number and atomic number of the reaction product, respectively; $\mathrm{n}$ - neutron; $b-\gamma$ photons, neutrons, charged particles, or helium nuclei), and the measurements of the reaction products [10]. Generally, NAA consists of three steps: irradiation with the thermal neutrons, measurements of gamma radiation after appropriate cooling time, and the interpretation of the gamma-ray spectra. The non-destructive phenomena of INAA (sample do not have to be digested prior to the analysis) make the technique attractive for chemical characterization of "difficult to digest" or "unique" materials. In 2011, INAA has been recognized as a primary method of measurements (PMMs) - the highest metrological range [11]. ICP-MS is a multielemental technique to determine low- and ultra-low-concentrations of elements. It is the most popular detection method, due to its precision, high sensitivity, low detection limits for majority of elements (LOD), wide linear dynamic range (up to eight orders of magnitude), and possibilities to determine isotopic ratios. Disadvantages and weaknesses of the ICP-MS detection are the occurrence of spectral and non-spectral interferences (e.g., accurate determination of ${ }^{99} \mathrm{Tc}$ may be difficult or even impossible, when stable ${ }^{99} \mathrm{Ru}$ and ${ }^{98} \mathrm{Mo}$ (via molecular ion ${ }^{98} \mathrm{Mo}{ }^{1} \mathrm{H}$ ) are present in sample solution) [12].
The Institute of Nuclear Chemistry and Technology (INCT) has been realizing the project financed by the National Centre for Research and Development (NCBR), entitled "Research and analysis of selected chemical aspects of the production and use of TRISO fuel in the HTGR nuclear reactor". As part of this project, analytical procedures have been developed that can be used to achieve control over the purity of substances used for fuel production and control over the fuel purity at each stage of its production [13]. Both ICP-MS (in solution mode and with laser evaporation of solid samples) and INAA were used in that project. This work aims to compare solution ICP-MS and INAA for analysis of selected substrates for TRISO fuel production. Also, the application of LA-ICP, MS, and INAA for the elemental analysis of chemically resistant protective layers was compared.

\section{Experimental}

\section{Samples and reagents}

The analysed samples (ascorbic acid, silicone oil, gasoline, extraction gasoline, ethanol, ammonia solution, hexamethylenetetramine, uranium oxides or salts) were obtained from the Sol-Gel Laboratory (INCT), where nuclear fuel is prepared $[14,15]$. In this work, only chemical analysis of two substances: ascorbic acid and silicone oil, is described in detail. Silicon carbide and pyrolytic graphite were obtained from Sigma Aldrich. Nitric acid (Chempur, Poland) was purified by a quartz sub-boiling distillation system. Suprapur grade hydrofluoric acid 40\% (Merck) was used for sample digestion. All other reagents used in this study $\left(30 \% \mathrm{H}_{2} \mathrm{O}_{2}\right.$ and $\mathrm{H}_{3} \mathrm{BO}_{3}$ (Chempur)) were of analytical grade. Further, high purity water (18 $\mathrm{M} \Omega \mathrm{cm}$ resistivity) was used (Millipore Co., Burlington, MA, USA) to prepare all solutions.

\section{Microwave-assisted total digestion and ICP-MS measurements}

Microwave-assisted digestion was applied for ascorbic acid and silicone oil using the Multiwave 3000 high-pressure microwave system (Anton Paar $\mathrm{GmbH}$ ). Then, known amounts of the materials (200-500 mg) were weighed into Teflon vessels. A single step process was realized for the decomposition of ascorbic acid using a mixture of concentrated $\mathrm{HNO}_{3}$ and $\mathrm{H}_{2} \mathrm{O}_{2}$. To digest silicone oil, first, a mixture of concentrated $\mathrm{HNO}_{3}, \mathrm{H}_{2} \mathrm{O}_{2}$ and $\mathrm{HF}$ was added. In second step, $18 \mathrm{~mL}$ of $4 \% \mathrm{H}_{3} \mathrm{BO}_{3}$ solution was introduced to the sample for the fluoride removal. The ELAN DRC II inductively coupled plasma quadrupole mass spectrometer (PerkinElmer) was used for ICP-MS measurements. The measurement parameters applied were radio frequency (RF) power $1000 \mathrm{~W}$, lens voltage $5.75 \mathrm{~V}$, plasma gas flow $13 \mathrm{~L} \cdot \mathrm{min}^{-1}$, auxiliary gas flow $1.2 \mathrm{~L} \cdot \mathrm{min}^{-1}$, nebulizer gas flow $0.89 \mathrm{~L} \cdot \mathrm{min}^{-1}$, measurement mode - standard, cones - Ni, Scott spray chamber, and cross flow nebulizer. 


\section{Laser ablation (LA)-ICP MS measurements}

In the case of SiC and pyrolytic graphite, LA was used for sampling of materials. LA system of LSX-500 Nd:YAG $266 \mathrm{~nm}$, max. energy $15 \mathrm{~mJ} / \mathrm{imp}$ (CETAC, USA) has been used for sample evaporation. The measurement parameters applied were RF power $1100 \mathrm{~W}$, lens voltage $5.75 \mathrm{~V}$, plasma gas flow $13 \mathrm{~L} \cdot \mathrm{min}^{-1}$, auxiliary gas flow $1.2 \mathrm{~L} \cdot \mathrm{min}^{-1}$, nebulizer gas flow $0.30 \mathrm{~L} \cdot \mathrm{min}^{-1}$, measurement mode - standard, and cones $-\mathrm{Ni}$.

\section{Neutron activation analysis}

Samples (silicon carbide, pyrolytic graphite, and ascorbic acid) were weighed into polyethylene (PE) capsules and sealed. The irradiation package consisted of samples, standards, and an empty capsule (blank) wrapped together in aluminium foil. Neutron activation was performed in the MARIA nuclear reactor (Świerk, Poland) with a thermal neutron flux of $10^{14} \mathrm{n} \cdot \mathrm{cm}^{-2} \cdot \mathrm{s}^{-1}$ for $30-50 \mathrm{~min}$. The cooling time was 3-7 days, depending on the activity of a given material. The HPGe well-type detector (Canberra), with relative efficiency $55 \%$ and energy resolution $2.1 \mathrm{keV}$ for the $1332 \mathrm{keV}$ peak of ${ }^{60} \mathrm{Co}$, coupled to Genie 2000 spectroscopy software (Canberra), has been used for gamma-ray measurements.

\section{Results and discussion}

Table 1 presents a comparison of selected properties important for INAA and ICP-MS, and which are important in inorganic trace analysis $[10,12]$. It can be seen that each technique can give reliable results and can be complementary applied in the characterization of nuclear samples.

Both of these analytical techniques, which are used for the determination of trace amounts of elements in a wide range of materials, are available in the Institute of Nuclear Chemistry and Technology.

\section{Comparison of results obtained with solution ICP-MS and INAA}

Table 2 contains results obtained for the ascorbic acid and silicone oil analysis. As can be seen, silicone oil is more contaminated by metals compared to ascorbic acid. In the case of NAA determination, due to short life of formed isotopes, it was not possible to determine such elements as: $\mathrm{Mn}$ $\left({ }^{54} \mathrm{Mn}, T_{1 / 2}-2.58 \mathrm{~h}\right), \mathrm{Cu}\left({ }^{64} \mathrm{Cu}, T_{1 / 2}-12.8 \mathrm{~h}\right), \mathrm{V}\left({ }^{52} \mathrm{~V}\right.$,

Table 1. Chosen features of ICP-MS and INAA used in elemental analysis of nuclear materials

\begin{tabular}{lcc}
\hline & ICP-MS & INAA \\
\hline Accuracy & Good & Excellent \\
Sensitivity & High & High \\
Matrix effect & Yes & No \\
Time response & Low-medium & Low \\
\hline
\end{tabular}

Table 2. Mean concentration of elements in ascorbic acid and silicone oil by ICP-MS and INAA $(n=3)$

\begin{tabular}{lcccc}
\hline \multirow{2}{*}{ Element } & \multicolumn{2}{c}{ Ascorbic acid } & \multicolumn{2}{c}{ Silicone oil } \\
\cline { 2 - 5 } & ICP-MS & NAA & ICP-MS & NAA \\
\hline $\mathrm{U}(\mathrm{ppb})$ & $<5$ & $<250$ & 152 & $<250$ \\
$\mathrm{Mn}(\mathrm{ppb})$ & 189 & n.d. & 1269 & n.d. \\
$\mathrm{Co}(\mathrm{ppb})$ & $<50$ & $<50$ & $<50$ & $<50$ \\
$\mathrm{Cd}(\mathrm{ppb})$ & $<50$ & n.d. & 1269 & n.d. \\
$\mathrm{Th}(\mathrm{ppb})$ & $<5$ & $<100$ & 1988 & 1780 \\
$\mathrm{Cu}(\mathrm{ppb})$ & $<250$ & n.d. & 1413 & n.d. \\
$\mathrm{Pb}(\mathrm{ppb})$ & 987 & n.d. & 1015 & n.d. \\
$\mathrm{V}(\mathrm{ppb})$ & $<180$ & n.d. & 205 & n.d. \\
$\mathrm{Sb}(\mathrm{ppb})$ & $<200$ & $<150$ & $<200$ & $<150$ \\
$\mathrm{Se}(\mathrm{ppb})$ & $<500$ & $<100$ & $<500$ & 72 \\
$\mathrm{Al}(\mathrm{ppm})$ & 17.9 & n.d. & 47.9 & n.d. \\
$\mathrm{Cr}(\mathrm{ppm})$ & $<2.5$ & $<1$ & $<2.5$ & 1.4 \\
$\mathrm{Ni}(\mathrm{ppm})$ & $<0.5$ & $<2.5$ & 2.5 & n.d. \\
$\mathrm{Fe}(\mathrm{ppm})$ & $<1$ & $<0.5$ & 186 & 150 \\
$\mathrm{Zn}(\mathrm{ppm})$ & 0.26 & 0.33 & 5.0 & 4.8 \\
\hline $\mathrm{n} . \mathrm{d} . \mathrm{not}$ & & & &
\end{tabular}

n.d., not determined.

$\left.T_{1 / 2}-3.75 \mathrm{~min}\right), \mathrm{Al}\left({ }^{28} \mathrm{Al}, T_{1 / 2}-2.2 \mathrm{~min}\right)$. Moreover, for lead analysis, its nuclear properties do not allow its content to be determined by NAA method (no gamma-ray emission).

From the data in Table 2, it can be seen that the two methods used have produced comparable results. However, if the uncertainty of measurement is compared, the results obtained by NAA show lower values of expanded uncertainty $(U)$. For ICP-MS, the expanded uncertainties $U(k=2)$ for the determined elements were calculated and were as follows: $5 \%$ for Th and $\mathrm{U} ; 10 \%$ for $\mathrm{Cd}, \mathrm{Co}, \mathrm{Cu}, \mathrm{Mn}, \mathrm{Ni}, \mathrm{Pb}, \mathrm{Sb}$, $\mathrm{V}$, and $\mathrm{Zn}$; and $15 \%$ for $\mathrm{Al}, \mathrm{Cr}$, and $\mathrm{Fe}$. In the case of INAA, the values of the expanded uncertainties $(k=2)$ were in the range 3-6\%.

\section{Comparison of results obtained with LA-ICP-MS and NAA}

In the case of materials with a great chemical resistance such as pyrolytic graphite or $\mathrm{SiC}$, it is difficult to find a suitable procedure to digest such materials for obtaining a complete transfer of analysed material to the solution. One of the decomposition methods for these materials is microwave-assisted digestion with a mixture of concentrated acids: $\mathrm{HNO}_{3}, \mathrm{H}_{2} \mathrm{SO}_{4}, \mathrm{HF}$, and other aggressive reagents. The possible limitations to microwave acid digestion of SiC or pyrolytic graphite are incomplete dissolution of sample, losses of volatile analytes, and the possibility of contamination. A fusion with alkaline mixtures can be an alternative to the acid digestion. Owing to the digestion of high salt content during the final fusion, it is necessary to dilute the sample (in ICP-MS measurement, the maximum salt content should not exceed $0.1 \%$ ) so that higher limits of detection are obtained. Moreover, the contamination of analysed material by the reagents can occur during the fusion procedure.

In the case of materials that are recognized as difficult to digest, application of LA-ICP-MS could 


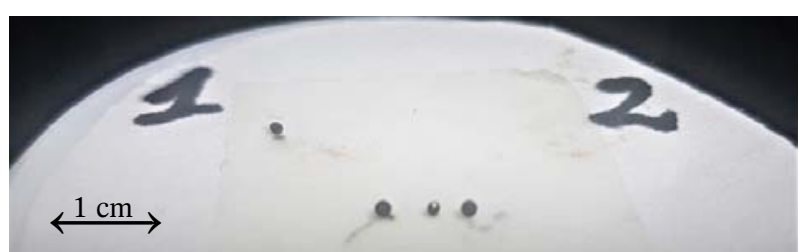

Fig. 1. LA stand with $\mathrm{SiC}$ (outer) and $\mathrm{Zr}$ (middle) spheres. Numbers allow positioning of the table in relation to the beam.

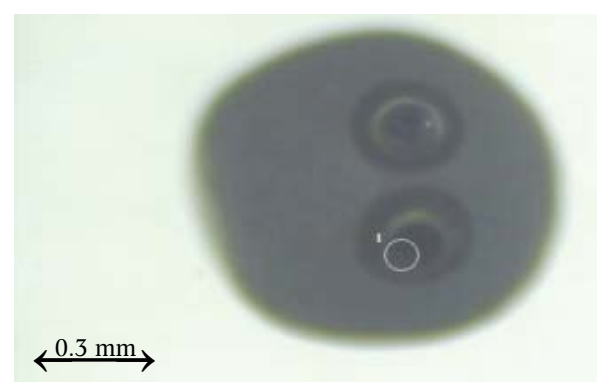

Fig. 2. Photo of a microsphere with spherical zirconia particle $\Phi=1 \mathrm{~mm}$ after laser evaporation.

be used as a complementary method for analysis. To ensure high nuclear safety of HTRs, highly chemical and thermal resistant protective layers (pyrolytic graphite and silicon carbide) are used. To avoid difficulties with total decomposition of these materials and analyse the composition of the obtained gaseous phase by the ICP-MS, the use of laser evaporation (LA) of the sample has been proposed. The coupling of LSX-500 laser from CETAC (USA) with ICP-MS ELAN DRC II spectrometer (PerkinElmer) was applied for the analysis of single spherical particle. Preliminary tests were carried out. The samples of 1-mm diameter with zirconium nucleus, covered with a layer of $\mathrm{PyC}$ and $\mathrm{SiC}$, were used for testing. A stand for analysis of single particles was made, i.e., a table for placing the grains (Fig. 1). To optimize the analysis conditions (maximum evaporation of individual components of the sample), the beam power and the duration of a single laser beam pulse were changed.

A laser beam pulse with a beam diameter of $150 \mu \mathrm{m}$ was directed onto the grains placed on the table. Pulse ablation was selected so that the depth of evaporated layer was $20 \mu \mathrm{m}$. Figure 2 shows a picture of the microsphere after laser evaporation of individual layers, and in Fig. 3 the obtained spec- tra (number of counts during sampling during profiling) are made available. The layers were evaporated in several steps, and evaporation was performed each time the hole depth was $20 \mu \mathrm{m}$.

Based on the spectra obtained, it can be concluded that the use of laser beam allows evaporation (decomposition) of protective layers of TRISO fuel microsphere and metal core. A laser of sufficient power penetrates the protective layers step by step, reaching the kernel. Initially, only the carbon signal is visible, then the silicon signal appears, and finally, in the mass spectrum, the signal from the fuel material (which is zirconium in this case) is visible. The following evaporation conditions are applied, namely laser beam diameter $-150 \mu \mathrm{m}$, frequency $20 \mathrm{~Hz}$, sampling interval $-15 \mathrm{~s}$, and measurement time $-10 \mathrm{~s}$.

Unfortunately, the lack of appropriate solid standards and certified reference materials dedicated to the LA-ICP-MS coupling may make it impossible to quantify selected elements in silicon carbide and pyrolytic graphite. In this work, NBS Glass 610 was used to conduct the screening analysis of both materials [16]. Table 3 shows the results obtained from the determination of contaminants in protective layers by NAA and LA-ICP-MS. Due to differences in the matrix (glass vs. SiC or pyrolytic graphite), different laser evaporation yields under the same conditions are obtained, and thus only semi-quantitative results can be given. For Eu, Hf, and Sc, it was not possible to determine these ele-

Table 3. Mean concentrations of elements as determined by NAA and LA ICP-MS in the analysed silicon carbide $(n=3)$ and pyrolytic graphite

\begin{tabular}{|c|c|c|}
\hline \multicolumn{3}{|c|}{ Silicon carbide } \\
\hline Element & NAA (ng/g) & LA-ICP-MS (ng/g) \\
\hline Co & 678 & $(950)$ \\
\hline $\mathrm{Eu}$ & 42 & n.d. \\
\hline $\mathrm{Fe}$ & 980 & (1390) \\
\hline Hf & 648 & n.d. \\
\hline $\mathrm{Sc}$ & 140 & n.d. \\
\hline \multicolumn{3}{|c|}{ Pyrolytic graphite } \\
\hline Element & NAA (ng/g) & LA-ICP-MS (ng/g) \\
\hline $\mathrm{Ag}$ & 320 & $(560)$ \\
\hline
\end{tabular}

n.d., not determined; (...) - semi quantitative, information results.
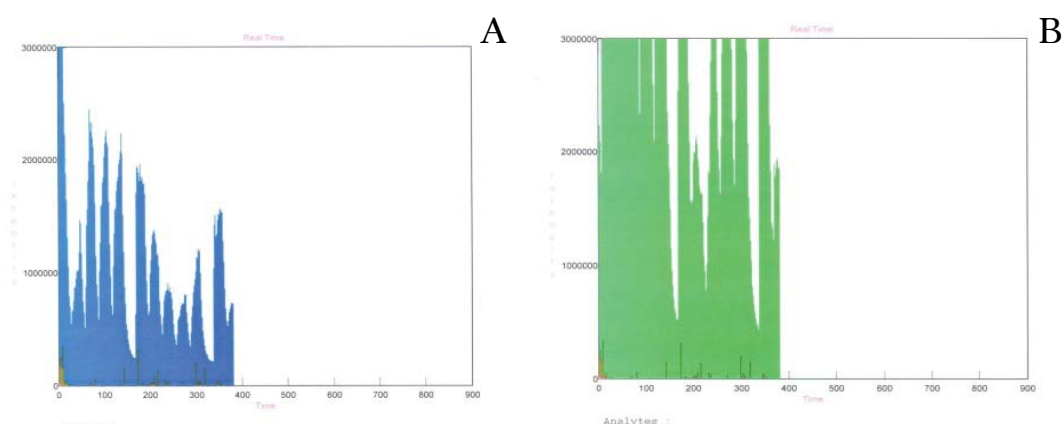

B

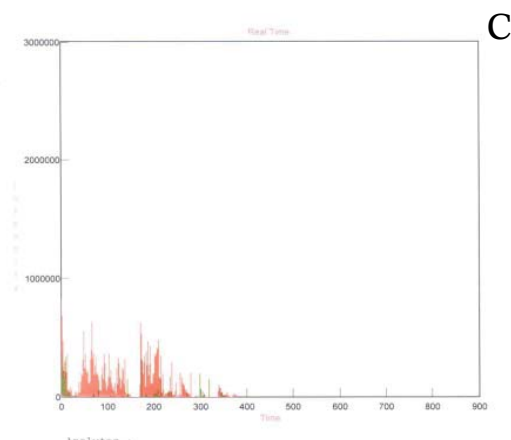

Fig. 3. Mass spectra of individual microsphere layers; LA-ICP-MS method: (A) C-12, (B) Si-28, and (C) Zr-90. 
ments in protective layers due to lack of certified or informative values in NBS610. It is therefore important to have a complementary method such as NAA, which will allow for quantitative results with low uncertainty values.

\section{Conclusions}

Both ICP-MS and INAA are reliable for elemental characterization of nuclear fuel and substances used for its production. Both may be suitable complementarily, which allows increase in the amount of the determined elements and controlling of the quality of the results obtained from the analysis. From the obtained results, it can be seen that silicon oil used in sol-gel fabrication could be a source of disturbing contaminants. Therefore, the influence of oil contaminants on fuel production should be investigated.

LA-ICP-MS was found to be useful for the semiquantitative analysis without any sample preparation. Despite its limitations, LA-ICP-MS can be used to study the composition of materials that are difficult to solubilize, among other things because of the short analysis time compared to INAA measurements. In this work, LA-ICP-MS was developed for the analysis of both single protective layers and single spherical zirconia particle. The measuring equipment was adapted to the requirements of the developed procedure: a target for irradiating single grains was made and qualitative analyses of microspheres were performed. In the case of analysis of commercially available $\mathrm{SiC}$ and pyrolytic graphite, it has been shown that the purity of silicon carbide must be carefully checked before it is used to produce protective layers. It has also been shown that there the possibility exists for the evaporation of the protective layers of pyrolytic graphite, silicon carbide, and the metal core. This suggests that it would be possible to study both the thickness of the protective layers and their purity when applied to the metallic kernel.

INAA, because of its unique possibilities, adhere to the Consultative Committee for Amount of Substance (CCQM) requirements for a PMM. The use of NAA allows the verification of analytical results obtained by other commonly used techniques, which is extremely important for ensuring and controlling the quality (quality control/quality assurance, QC/QA) of analytical results in nuclear laboratories. Therefore, this method should be included for testing the purity of materials used in fuel production.

Acknowledgments. This work is one portion of the studies in the strategic Polish programme of scientific research and development work "Social and economic development of Poland in the conditions of globalizing markets GOSPOSTRATEG", which is part of "Preparation of legal, organizational and technical instruments for the HTR implementation" financed by the National Centre for Research and Development (NCBR) in Poland.

\section{References}

1. International Atomic Energy Agency. (2010). High temperature gas cooled reactor fuels and materials. Vienna: IAEA. (IAEA-TECDOC-1645).

2. Quade, R. N., \& McMain, A. T. (1975). Hydrogen production with a high-temperature gas-cooled reactor (HTGR). In T. N. Veziroglu (Ed.), Hydrogen energy (pp. 137-154). New York: Plenum Press.

3. Chao Fang, C., Morris, R., \& Li, F. (2017). Safety features of high temperature gas cooled reactor. Sci. Technol. Nucl. Install., 2017, art. ID 9160971. DOI: 10.1155/2017/9160971.

4. Verfondern, K., Nabielek, H., \& Kendall, J. M. (2007). Coated particle fuel for high temperature gas cooled reactors. Nucl. Eng. Technol., 39, 603-616. DOI: 10.5516/NET.2007.39.5.603.

5. De Souza, A. L., Cotrim, M. E. B., \& Pires, M. A. F. (2013). An overview of spectrometric techniques and sample preparation for the determination of impurities in uranium nuclear fuel grade. Microchem. J., 106, 194-201. DOI: 10.1016/j.microc.2012.06.015.

6. Sadikov, I. I., Rakhimov, A. V., Salimov, M. I., Zinov'ev, V. G., Mukhamedshina, N. M. F., \& Tashimova, A. (2009). Neutron activation analysis of pure uranium: Preconcentration of impurity elements. J. Radioanal. Nucl. Chem., 280, 489-493. DOI: 10.1007/s10967-008-7389-y.

7. Oliveira Junior, O. P., \& Sarkis, J. E. S. (2002). Determination of impurities in uranium oxide by inductively coupled plasma mass spectrometry (ICPMS) by the matrix matching method. J. Radioanal. Nucl. Chem., 254, 519-526. DOI: 10.1023/A:1021642122066.

8. Bürger, S., Riciputi, L. R., \& Bostick, D. A. (2007). Determination of impurities in uranium matrices by time-of-flight ICP-MS using matrix-matched method. J. Radioanal. Nucl. Chem., 274, 491-505. DOI: 10.1007/s10967-006-6930-0.

9. Saha, A., Kumari, K., Deb, S. B., \& Saxena, M. K. (2021). Determination of critical trace impurities in "uranium silicide dispersed in aluminium" nuclear fuel by inductively coupled plasma mass spectrometry (ICP-MS). J. Anal. At. Spectrom., 36, 561-569. DOI: 10.1039/D0JA00391C.

10. Bode, P. (1996). Instrumental and organizational aspects of a neutron activation analysis laboratory. Delft, The Netherlands: Delft University of Technology.

11. Greenberg, R. R., Bode, P., \& De Nadai Fernandes, E. A. (2011). Neutron activation analysis: A primary method of measurement. Spectroc. Acta Pt. B-Atom. Spectr., 66(3/4), 193-241. DOI: 10.1016/j. sab.2010.12.011.

12. Ammann, A. A. (2007). Inductively coupled plasma mass spectrometry (ICP MS): a versatile tool. J. Mass Spectrom., 42, 419-427. DOI: 10.1002/jms.1206.

13. Chajduk, E., Kalbarczyk, P., Dudek, J., Pyszynska, M., Bojanowska-Czajka, A., \& Samczyński, Z. (2020). Development of analytical procedures for chemical characterization of substrates for the production of TRISO coated particles as nuclear fuel in high temperature gas-cooled reactors. Sustainability, 12(17), 7221-7234. DOI: 10.3390/su12177221.

14. Brykała, M., Rogowski, M., Wawszczak, D., Olczak, T., \& Smoliński, T. (2020). Microspheres and pellets 
of $\mathrm{UO}_{2}$ prepared via ADU by complex sol-gel process and ICHTJ process. Arch. Metall. Mater., 65(4), 1397-1404. DOI: 10.24425/amm.2020.133706.

15. Deptuła, A., Brykała, M., Rogowski, M., Smoliński, T., Olczak, T., Łada, W., Wawszczak, D., Chmielewski, A., \& Goretta, K. C. (2014). Fabrication of uranium dioxide microspheres by classic and novel sol-gel processes. MRS Online Proceedings Library, 1683, 64-69. https://doi.org/10.1557/opl.2014.672.

16. National Institute of Standards and Technology (2012). Certificate of Analysis. Standard Reference Material 610. Available from https://www-s.nist.gov/ srmors/certificates/610.pdf. 